Hirnmetastasen beim Bronchialkarzinom

\section{Gamma Knife als Alternative zur Resektion}

Flannery TW et al. Long-term survival in patients with synchronous, solitary brain metastasis from non-small-cell lung cancer treated with radiosurgery. Int J Radiat Oncol Biol Phys 2008;72:19-23

\section{Hintergrund}

Das Bronchialkarzinom ist für etwa 50\% aller Hirnmetastasen verantwortlich [1]. Zwei von drei randomisierten Studien kamen zu dem Schluss, dass die Resektion singulärer oder solitärer Hirnmetastasen, gefolgt von einer Ganzhirnbestrahlung, der alleinigen Ganzhirnbestrahlung bezüglich der Überlebenszeit überlegen ist. Eine dritte Studie belegte den Wert der Operation nicht. Zahlreiche retrospektive Analysen sprechen ebenfalls für die Resektion singulärer oder solitärer Hirnmetastasen. Durch die Resektion des Primärtumors und der solitären Hirnmetastase können 5-JahresÜberlebensraten von $11-21 \%$ erreicht werden $[1,2]$.

\section{Fragestellung}

Mit der stereotaktischen Gamma-KnifeBestrahlung („Gamma Knife Stereotactic Radiosurgery“, GKSRS) steht eine weitere alternative Möglichkeit zur Behandlung solitärer Hirnmetastasen - die in

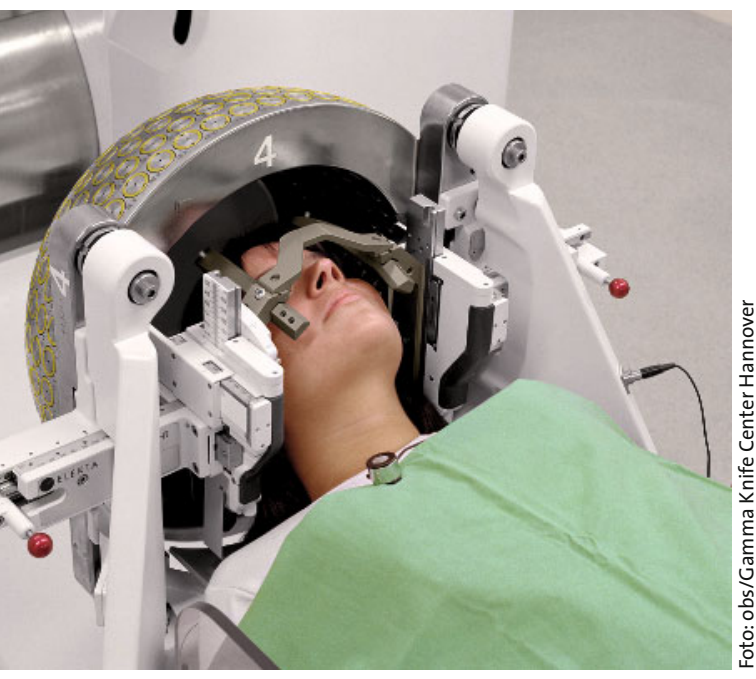

Stereotaktische Bestrahlung mit dem Gamma Knife. der Regel nicht größer als $3 \mathrm{~cm}$ sein sollten - zur Verfügung. Eine amerikanische retrospektive Analyse legte Daten zur GKSRS bei Patienten mit einem nicht kleinzelligen Bronchialkarzinom (NSCLC) und einer synchronen, solitären Hirnmetastase vor.

\section{Methodik}

42 NSCLC-Patienten (medianes Alter: 58 Jahre; medianer Karnofsky-Index = 90) wurden in die Studie eingeschlossen. Die Größe der singulären Hirnmetastasen lag zwischen 0,5 und $3,5 \mathrm{~cm}$ (Median: $1,5 \mathrm{~cm}$ ).

Die Behandlung erfolgte innerhalb von drei Monaten nach der Primärdiagnose. Der Median der 50\%-Isodose für die GKSRS war 18 Gy. 33 der 42 Patienten erhielten zusätzlich eine Ganzschädelbestrahlung, davon zwölf erst nach dem Abschluss der Gesamtbehandlung zum Zeitpunkt einer neurologischen Befundprogression.

26 von 42 Patienten (62\%) erhielten eine definitive thorakale Therapie (Chemoradiotherapie, Operation oder trimodale Therapie). 20 dieser 26 Patienten hatten einen Karnofsky-Index $\geq 90 \%$. 16 Patienten erhielten keine definitive thorakale Therapie (alleinige Chemotherapie bzw. palliative Radiotherapie); vier von ihnen hatten einen KarnofskyIndex $\geq 90$.

\section{Ergebnisse}

Für alle 42 Patienten wurden eine mediane Überlebenszeit von 18 Monaten und eine 5-Jahres-Überlebensrate von 21\% erreicht. 20\% der Patienten mit Erkrankungsprogression hatten eine neurologische Progression. Bei einem Patienten musste eine symptomatische zerebrale Radionekrose operativ entfernt werden.
In der multivariaten Analyse waren eine definitive thorakale Therapie (26,4 vs. 13,1 Monate; 5-Jahres-Überlebensrate 34,6 vs. $0 \%$; $\mathrm{p}<0,0001)$ und ein Karnofsky-Index $\geq 90$ (27,8 vs. 13,1 Monate, $\mathrm{p}<0,0001)$ signifikante Prädiktoren für ein längeres Überleben. Darüber hinaus zeigte sich ein Trend für ein längeres Überleben, wenn keine mediastinalen Lymphknotenmetastasen vorlagen (N0/N1 vs. N2/N3: 21,1 vs. 14,1 Monate; $\mathrm{p}=0,08)$.

\section{Kommentar}

Priv.-Doz. Dr. med.

Martin Kohlhäufl, Stuttgart-Gerlingen

Diese retrospektive Studie zeigt, dass Patienten in gutem Allgemeinzustand mit einer solitären, synchron zur Primärerkrankung diagnostizierten Hirnmetastase kurativ behandelt werden können - vorausgesetzt, eine adäquate Therapie der Primärerkrankung ist möglich.

In dieser Studie erfolgte die Behandlung solitärer Hirnmetastasen durch eine GKSRS. Patienten, deren Hirnmetastase mit GKSRS behandelt worden war und die eine definitive thorakale Therapie erhalten hatten, erreichten eine 5-Jahres-Überlebensrate von $34,6 \%$.

\section{Fazit}

Mit der GKSRS steht eine effektive alternative Möglichkeit zur Behandlung solitärer Hirnmetastasen $(<3 \mathrm{~cm})$ zur Verfügung. Somit kann eine GKSRS für Patienten mit einer synchronen solitären Hirnmetastasen und der Möglichkeit zur definitiven Behandlung des Primärtumors, möglichst ergänzt um eine Ganzschädelbestrahlung, empfohlen werden.

\section{Literatur}

1. Neuroonkologische Arbeitsgemeinschaft (NOA) in der Deutschen Krebsgesellschaft (2008) Leitlinie: Solide Hirnmetastasen. www.neuroonkologie. de

2. Hart MG et al. Surgical resection and whole brain radiation therapy versus whole brain radiation therapy alone for single brain metastases. Cochrane Database Syst Rev 2004, 4:CD 003292 